\title{
Exact equation of state for ideal relativistic quantum gases
}

\author{
Frithjof Karsch \\ Department of Theoretical Physics, University of Bielefeld, 48 Bielefeld 1, West Germany \\ David E. Miller \\ Center for Interdisciplinary Research and Department of Theoretical Physics, University of Bielefeld, West Germany
}

(Received 7 September 1979)

\begin{abstract}
The use of analytical methods yields the fugacity of the ideal relativistic quantum gases in terms of the particle densities, pressures, and energy densities, which we compute numerically. The exact equation of state in the thermodynamic limit arising from these solutions may be computed and compared with their respective series expansions.
\end{abstract}

\section{INTRODUCTION}

The problems relating to a manifestly covariant formulation of equilibrium thermodynamics and statistical mechanics have been widely discussed in the literature. ${ }^{1}$ Within this framework one can derive the invariant phase-space measure for the ideal relativistic quantum gases, which was used by Touscheck ${ }^{2}$ in his covariant formulation of relativistic statistical mechanics. We shall show in this paper how this structure yields a proper basis for the discussion of the ideal relativistic gases (IRG) whose cluster structure ${ }^{3}$ and equation of state $^{4,5}$ have been previously investigated. Although the qualitative behavior of the thermodynamical quantities of the IRG is quite clear, it may well be of interest to study their detailed structure in order to compare it with the corresponding series expansions. The understanding gained through this comparison may then be informative as a starting point for the interacting system, for which the IRG serve as the first simplified model usable even in several branches of actual physical research. The nonrelativistic and ultrarelativistic limits are often too crude approximations for relativistic massive systems since they neglect the temperature dependence present in the relativistic cluster coefficients.

The thermodynamical potential $\Omega(\beta, V, A)$ is related to the grand partition function $\Xi(\beta, V, A)$ in covariant form as a function, respectively, of the inverse temperature $\beta$, the volume $V$, and the fugacity $A$. This relationship leads to the known for $\mathrm{m}^{3}$

$$
\Omega(\beta, V, A)=-\beta^{-1} \eta \int d \sigma \ln \left[1+\eta A \exp \left(-\beta_{\mu} p^{\mu}\right)\right](1 \mathrm{a})
$$

with $\eta= \pm 1$ for Fermi or Bose statistics and $d \sigma$ the phase-space measure:

$$
d \sigma=2 V_{\mu} p^{\mu} \theta\left(p_{0}\right) \delta\left(p^{2}-m^{2}\right) \frac{d^{4} p}{(2 \pi)^{3}} .
$$

The statement of the first and second laws of thermodynamics implies directly the usual demands on the non-negativity of the specific heat and the compressibility. 6 Particularly interesting for our further investigation is the relationship involving the particle number $N$. Analogous with the non-negativity of the specific heat and compressibility it may be noted that the external conditions on the entropy demand that

$$
\left(\frac{\partial A}{\partial N}\right)_{B, V} \geq 0 \text {. }
$$

We shall see later how the case of strict equality relates to the large fluctuations yielding the BoseEinstein condensation. The condition in (2) relates directly to the particle mean square fluctuations ${ }^{6}$ $(\Delta N)^{2}$, which is clearly non-negative, so that we find

$$
\overline{(\Delta N)^{2}}=\beta^{-1}\left(\frac{\partial N}{\partial \mu}\right)_{B, V},
$$

where $\mu$ is the relativistic chemical potential by $\beta^{-1} \ln A$. The importance of these relationships will become clear from our calculations on the IRG of the following sections.

We shall evaluate the thermodynamical functions; in particular the pressure $p$, the particle density $n$, and the energy density $\epsilon$ from the use of the thermodynamic potential $\Omega$ in Eq. (1a) in the grand canonical ensemble. We can readily calculate these quantities by carrying out the necessary operations as given by

$$
\begin{aligned}
& p=-\frac{1}{V} \Omega(\beta, V, A), \\
& n=-\frac{A}{V} \frac{\partial}{\partial A}[\beta \Omega(\beta, V, A)], \\
& \epsilon=\frac{1}{V} \frac{\partial}{\partial \beta}[\beta \Omega(\beta, V, A)] .
\end{aligned}
$$

The Eqs. (4a)-(4c) can be solved for $A$ as was first carried out by Leonard ${ }^{7}$ for the nonrelativistic

(c) 1980 The American Physical Society 
quantum gases and then by Nieto ${ }^{5}$ for the ideal relativistic quantum gases using an analytic method based upon the theory of singular integral equations. ${ }^{8}$ After combining pairwise these equations resolved for $A$, one arrives at the exact equations of state in integral form. These equations will be analyzed in the thermodynamic limit using various approximate (virial) types of expansions as have been employed for a long time in the classical interacting gas theories.

In this paper we shall discuss the thermodynamical properties of the IRG. In Sec. II we shall develop the mathe matical techniques for the computation of the exact fugacity equations for both the Fermi and Bose systems which are compared with a relativistic Boltzmann gas. 4 The following section uses these results in the thermodynamic limit for the exact equation of state of the IRG for these cases as has been remarked by Balescu. ${ }^{9}$ We shall discuss in Sec. IV the virial expansion of the equation of state, which is gotten from the cluster decomposition of the grand partition function and is compared for the first few orders with the numerical results of exact solutions. Section $V$ contains our conclusions with some remarks about the possible generalizations.

\section{INVERSION OF THE FUGACITY EQUATIONS}

Through the use of Eq. (1a) we are able to calculate the thermodynamical quantities $p, n$, and $\epsilon$ from (4a)-(4c) for the IRG from $\Omega$ :

$$
\begin{aligned}
p= & \frac{A m^{4}}{6 \pi^{2}} g \int_{1}^{\infty} d t\left(t^{2}-1\right)^{3 / 2} \frac{1}{e^{m b t}+\eta A} \\
& +\left\{\begin{array}{l}
0 \\
0 \\
-\frac{1}{V \beta} \ln \left(1-A e^{-m \beta}\right)
\end{array}\right. \\
n= & \frac{A m^{3}}{2 \pi^{2}} g \int_{1}^{\infty} d t t\left(t^{2}-1\right)^{1 / 2} \frac{1}{e^{m B t}+\eta A} \\
& +\left\{\begin{array}{l}
0 \\
0 \\
\frac{1}{V} \frac{A}{e^{m B}-A}
\end{array}\right. \\
\epsilon= & \frac{A m^{4}}{2 \pi^{2}} g \int_{1}^{\infty} d t t^{2}\left(t^{2}-1\right)^{1 / 2} \frac{1}{e^{m B t}+\eta A} \\
& +\left\{\begin{array}{l}
0 \\
0 \\
\frac{1}{V} \frac{m A}{e^{m B}-A}
\end{array}\right.
\end{aligned}
$$

$g$ is the usual statistical factor and in all these equations $[(5 a)-(5 c)]$ the upper term is for the fermions $(\eta=+1)$, the lower one is for the bosons $(\eta=-1)$, while the middle one is for the classical gas $(\eta=0)$.

When we perform the substitution $y=\exp [m \beta(t$ $-1)$ ] into these equations, we find the following integrals of the Cauchy type ${ }^{8}$ :

$$
\begin{aligned}
& \beta p=z \Lambda^{-3} \int_{1}^{\infty} d y \frac{f_{p}(y)}{y(y+\eta z)}+\left\{\begin{array}{l}
0 \\
0 \\
1 \\
-\frac{1}{V} \ln (1-z)
\end{array},\right. \\
& n=z \Lambda^{-3} \int_{1}^{\infty} d y \frac{f_{n}(y)}{y(y+\eta z)}+\left\{\begin{array}{l}
0 \\
0 \\
\frac{1}{V} \frac{z}{1-z}
\end{array}\right. \\
& \beta \epsilon=z \Lambda^{-3} \int_{1}^{\infty} d y \frac{f_{\varepsilon}(y)}{y(y+\eta z)}+\left\{\begin{array}{l}
0 \\
0 \\
\frac{1}{V} \frac{m \beta z}{1-z} .
\end{array}\right.
\end{aligned}
$$

The letter $z=A / A_{c}$ with $A_{c}=\exp (m \beta)$ is the normalized relativistic fugacity, which coincides with the nonrelativistic fugacity. The so called" "optical wavelength" $\Lambda$ is such that $\Lambda^{3} g$ is given by $2 \pi^{2} \beta^{3}$. The functions $f_{h}(y)$ with $h$ representing $p, n, \epsilon$ are given by

$$
\begin{aligned}
& f_{p}(y)=[\ln y(\ln y+2 m \beta)]^{3 / 2} / 3, \\
& f_{n}(y)=(\ln y+m \beta)[\ln y(\ln y+2 m \beta)]^{1 / 2}, \\
& f_{\epsilon}(y)=(\ln y+m \beta)^{2}[\ln y(\ln y+2 m \beta)]^{1 / 2} .
\end{aligned}
$$

The basic problem for ideal quantum gases concerns the solution ${ }^{5,7}$ of Eqs. (6a)-(6c) for $z$ in order to find the fugacity as a function of $p, n$, or $\epsilon$, respectively. For the classical gas this problem is trivial because the fugacity then appears only as a multiplicative factor. However, for the quantum gases the relationship to the fugacity is much more complicated. Its solution was first obtained by Leonard ${ }^{7}$ for the nonrelativistic quantum gases, for which he interpreted $z$ as a complex variable in order to use the Hilbert proble ${ }^{8}$ for the inversion of Eqs. (6a)-(6c). Afterwards, the same methods were used for the ideal relativistic quantum gases ${ }^{5}$ as well as for the relativistic gases with a given hadronic mass spectrum ${ }^{10}$ since in all these cases the structure of the thermodynamical functions is quite similar and differs only through the stated form of the involved functions $f_{h}$.

Through the examination of the asymptotic behavior of the thermodynamical functions $p, n, \epsilon$ on the branch cut $(-\infty,-1]$ for fermions and $[1, \infty)$ for 
bosons, which is induced by the Cauchy integrals, one gets the essential information for the solution of the Hilbert problem. The mathematical details are given in the cited literature.$^{5,7}$ For the normalized fugacity $z$ of the IRG it is then found

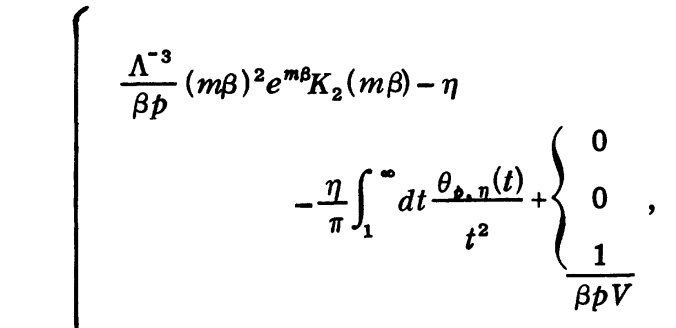

$$
\begin{aligned}
& \frac{1}{z}=\left\{\frac{\Lambda^{-3}}{n}(m \beta)^{2} e^{m \beta} K_{2}(m \beta)-\eta\right. \\
& -\frac{\eta}{\pi} \int_{1}^{\infty} d t \frac{\theta_{n, \eta}(t)}{t^{2}}+\left\{\begin{array}{c}
0 \\
0 \\
\frac{1}{n V}
\end{array},\right. \\
& \frac{\Lambda^{-3}}{\beta \epsilon}(m \beta)^{2} e^{m \beta}\left[m \beta K_{1}(m \beta)+3 K_{2}(m \beta)\right]-\eta \\
& -\frac{\eta}{\pi} \int_{1}^{\infty} d t \frac{\theta_{\epsilon, n}(t)}{t^{2}}+\left\{\begin{array}{c}
0 \\
0 \\
\frac{m}{\epsilon V}
\end{array}\right.
\end{aligned}
$$

Here $K_{i}(x)$ stands for the modified Bessel functions of the second kind. ${ }^{11}$ If we define the principal value integrals ${ }^{8}$ for the functions

$$
F_{h}(t)=t \int_{1}^{\infty} d y \frac{f_{h}(y)}{y(y-t)}, \quad h=p, n, \epsilon ; \quad t \in[1, \infty),
$$

the functions $\theta_{h,+1}(t)$ for fermions are given as

$$
\theta_{h,+1}(t)=\arctan \left(\frac{\pi f_{h}(t)}{F_{h}(t)+\Lambda^{3} \tilde{h}}\right), \quad \tilde{h}=\beta p, n, \beta \epsilon
$$

where the branch of the arctangent is chosen such that

$$
\begin{aligned}
& \lim _{t \rightarrow \infty} \theta_{n,+1}(t)=0, \\
& \lim _{t \rightarrow 1} \theta_{n_{0}+1}(t)=-\pi .
\end{aligned}
$$

For bosons the functions $\theta_{n,-1}(t)$ contain additional condensation terms

$$
\begin{aligned}
& \theta_{p,-1}(t)=\arctan \left(\frac{\pi f_{0}(t)}{F_{p}(t)-\left(\Lambda^{3} / V\right) \ln (t-1)-\Lambda^{3} \beta p}\right), \\
& \theta_{n,-1}(t)=\arctan \left(\frac{\pi f_{n}(t)}{F_{n}(t)-\left(\Lambda^{3} / V\right)(t / t-1)-\Lambda^{3} n}\right),
\end{aligned}
$$

$$
\theta_{\epsilon,-1}(t)=\arctan \left(\frac{\pi f_{\epsilon}(t)}{F_{\epsilon}(t)-\left(\Lambda^{3} / V\right)[m \beta t /(t-1)]-\Lambda^{3} \beta \epsilon}\right) .
$$

The limits (11a) and (11b) are reached in the thermodynamic limit for those cases where Bose condensation has not yet taken place. ${ }^{5}$ For completeness we define for the classical gas

$$
\theta_{h, 0}(t) \equiv 0, h=p, n, \epsilon .
$$

Obviously, in the thermodynamic limit the Eqs. (9), (10), (12), and (13) can be summarized in the following formula:

$$
\theta_{h, \eta}(t)=\eta^{2} \arctan \left(\frac{\pi f_{h}(t)}{F_{h}(t)+\eta \Lambda^{3} \bar{h}}\right) .
$$

Before we look at the numerical results for the fugacity in terms of the three different thermodynamical parameters let us discuss Eqs. (8) in a bit more detail. Since the functions $\theta_{h, \eta}(t)$ are bounded by $-\pi$ from below and 0 from above, the corresponding integrals in $\mathrm{Eq}$. (8) are limited by the same bounds. Therefore, at low pressures or, respectively, at low densities the statistically dependent terms in Eq. (8) become irrelevant and only the first term characterizes the behavior of the fugacity. Thus the relativistic quantum gases are in this case well described by the corresponding relativistic Boltzmann gas.

For the parametrization of the numerical results it is convenient to introduce the function

$$
L(m, \beta)=\Lambda^{-3}(m \beta)^{2} e^{m \beta} K_{2}(m \beta)
$$

which has the property

$$
\begin{aligned}
& \lim _{m \beta \rightarrow 0} L(m, \beta)=2 \Lambda^{-3}, \\
& \lim _{m \beta \rightarrow \infty} L(m, \beta)=g \lambda^{-3},
\end{aligned}
$$

where $\lambda=(2 \pi \beta / m)^{1 / 2}$ is the thermal wavelength. One should notice that $L(m, \beta)$ is just the particle density of a relativistic Boltzmann gas with $z$ equal to one.

Figure 1 shows the normalized fugacity $z$ of the IRG as a function of $L^{-1}(m, \beta) m$ in the thermodynamic limit. Due to the chosen parametrization, the fugacity of a Boltzmann gas (broken line) is now in this case independent of $m$ and $\beta$, while it depends only on the product $m \beta$ for the relativistic quantum gases. Figure 1 clearly reflects the stability condition (2) mentioned in the Introduction.

The cases $m \beta=0.01$ and $m \beta=5$ correspond to the two limiting cases of an ultrarelativistic and nonrelativistic gas. This correspondence can be seen, if one compares the condensation densities $n_{c}$ of a Bose gas, for which one has in both limits ${ }^{5}$ 

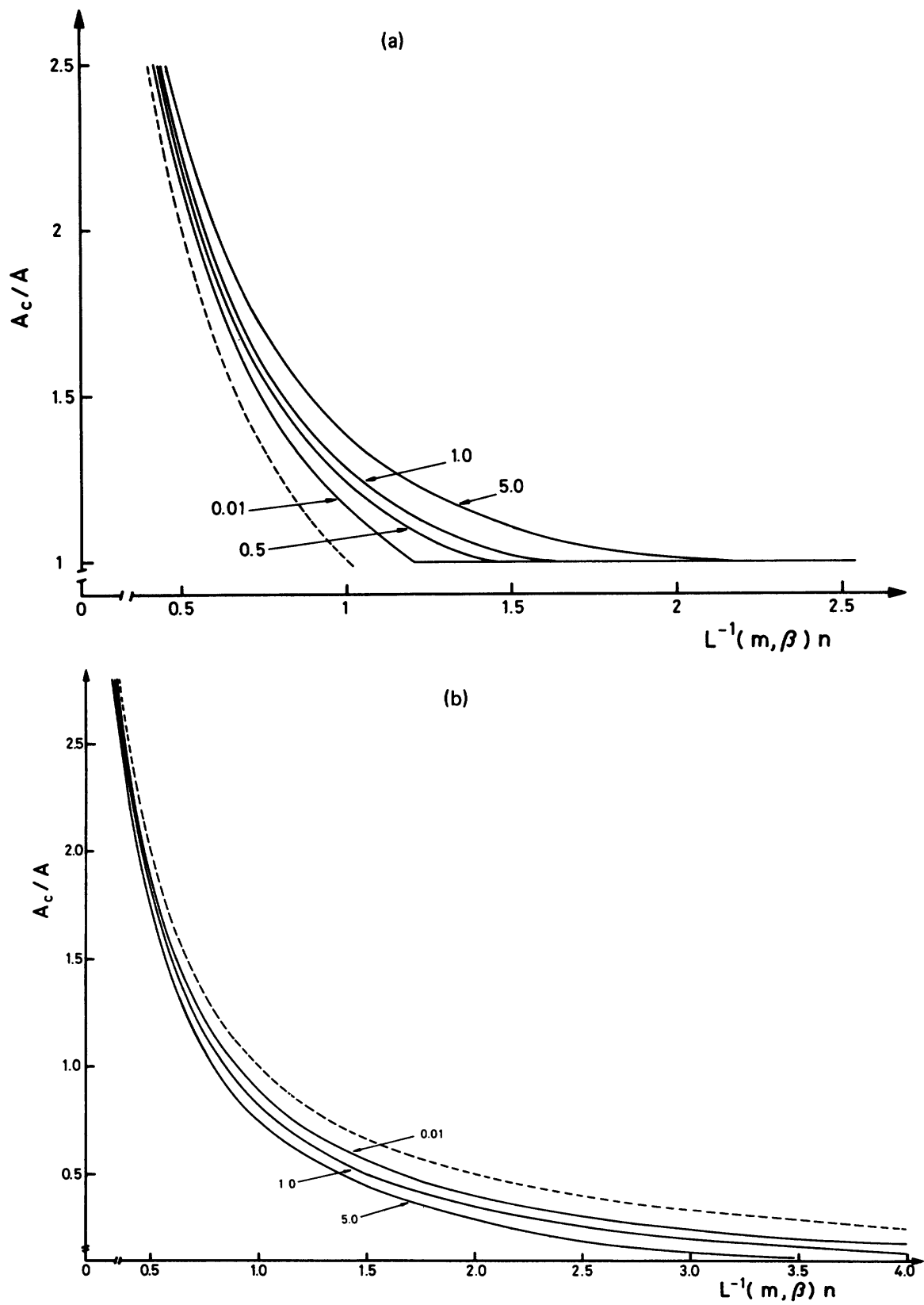

FIG. 1. Inverse normalized fugacity versus $L^{-1}(m, \beta) n$ for (a) Bose and (b) Fermi gases. The dependence on $m \beta$ for the quantum gases $(-)$ is compared with the classical Boltzmann gas (---).

$$
\begin{aligned}
& \lim _{m \beta \rightarrow 0} n_{c}=2 \Lambda^{-3} \zeta(3)=(1.202) 2 \Lambda^{-3}, \\
& \lim _{m \beta \rightarrow \infty} n_{c}=\lambda^{-3} \zeta\left(\frac{3}{2}\right)=2.612 \lambda^{-3},
\end{aligned}
$$

with those of Fig. 1(a). In order to get a feeling for the densities where Bose condensation occurs, the scaling factor $L^{-1}(m, \beta)$ is given in Table $I$ for the special case of a pion gas with mass $m_{\pi}=140 \mathrm{MeV}$.

For the special case $m \beta=0.5$ the behavior of $z$ as a function of the three thermodynamic quantities $\beta p, n$, and $\frac{1}{3} \beta \epsilon$ is compared in Fig. 2. The fact that the relative order of the curves in Fig. 2(a) (Bose gas) and Fig. 2(b) (Fermi gas) is interchanged clearly expresses the attractive (Bose) and repulsive (Fermi) nature of the different kinds of statistic.

\section{EQUATIONS OF STATE}

Once one has derived the fugacity $z$ as a function of $p, n$, and $\epsilon$, there is no problem in getting the 
TABLE I. Scaling factor $L^{-1}(m \beta)$ for the case of a pion gas.

\begin{tabular}{ccccc}
\hline \hline$m_{\pi} \beta$ & 0.01 & 0.5 & 1 & 5 \\
\hline$L^{-1}\left(m_{\pi}, \beta\right)\left(\mathrm{GeV}^{-3}\right)$ & $3.56 \times 10^{-3}$ & $2.89 \times 10^{2}$ & $1.63 \times 10^{3}$ & $4.57 \times 10^{4}$ \\
\hline \hline
\end{tabular}
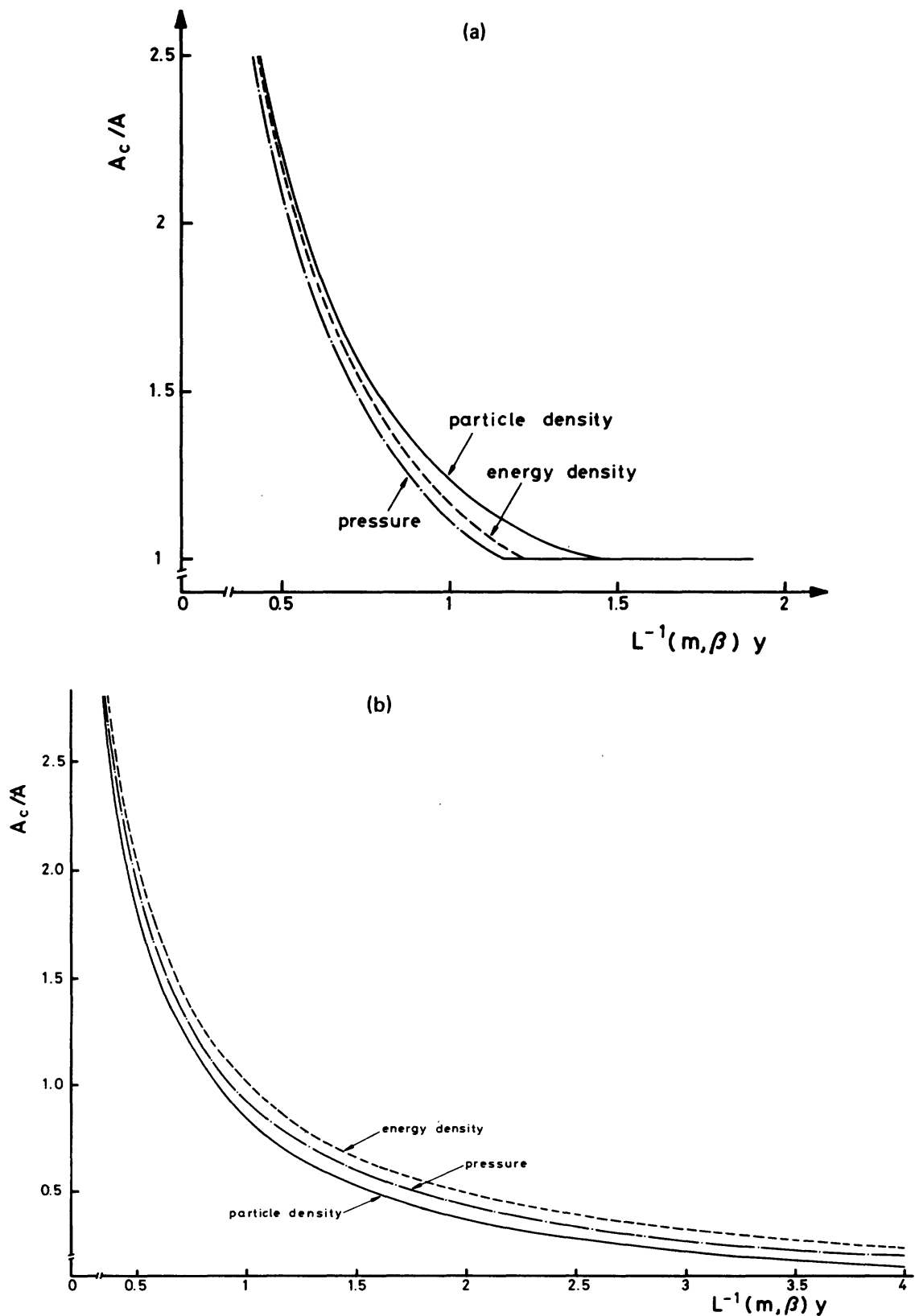

FIG. 2. Inverse normalized fugacity versus $L^{-1}(m, \beta) y, y=p, n, \frac{1}{3} \beta \epsilon$, for (a) Bose and (b) Fermi gas for the special value $m \beta=0.5$. 
exact equations of state for relativistic gases through the combination of any two of these three relations. In the thermodynamic limit we get, therefore, the three equations of state:

$$
\begin{aligned}
\left(\frac{1}{n}-\frac{1}{\beta p}\right) L(m, \beta) & =\frac{\eta}{\pi} \int_{1}^{\infty} d t \frac{\theta_{n, \eta}(t)-\theta_{\beta_{n} n}(t)}{t^{2}}, \\
\left(\frac{1}{\beta p}-\frac{3}{\beta \epsilon}\right) L(m, \beta) & -\frac{\Lambda^{-3}}{\beta p}(m \beta)^{3} e^{m \beta} K_{1}(m \beta) \\
& =\frac{\eta}{\pi} \int_{1}^{\infty} d t \frac{\theta_{n_{n}, n}(t)-\theta_{\epsilon, n}(t)}{t^{2}}, \\
\left(\frac{1}{n}-\frac{3}{\beta \epsilon}\right) L(m, \beta) & -\frac{\Lambda^{-3}}{\beta \epsilon}(m \beta)^{3} e^{m \beta} K_{1}(m \beta) \\
& =\frac{\eta}{\pi} \int_{1}^{\infty} d t \frac{\theta_{n, n}(t)-\theta_{\epsilon, n}(t)}{t^{2}} .
\end{aligned}
$$

It is clear from the structure of Eqs. (18) that the main deviation of the relativistic quantum gases from the behavior of a relativistic Boltzmann gas is contained in the right-hand side of the se equations. For a Boltzmann gas $(\eta=0) \mathrm{Eq}$. (18a) yields the ideal gas equation $\beta p / n=1$, which is compared in Fig. 3 with the behavior of the relativistic quantum gases. Obviously, the deviation from the classical behavior becomes smaller when the parameter $m \beta$ becomes smaller. In Fig. 3 the condensation points are marked by an arrow. For $m \beta=5$ the condensation would occur at $L^{-1}(m, \beta) n=2.14$. However, it must be remarked that the crossing of the curves is only due to the chosen units (see Table I).
We have also computed the kinetic energy per particle $(\epsilon / n-m)$ times the inverse temperature $\beta$. The result is shown in Fig. 4 for Bose [Fig. 4(a)] and for Fermi [Fig. 4(b)] gases. Again the condensation points are marked by an arrow. The starting point of the curves at $L^{-1}(m, \beta) n=0$ can be seen to range between the two limits, 1.5 for a nonrelativistic gas and 3 for an ultrarelativistic gas.

\section{SERIES EXPANSIONS}

In this section we compare the exact solutions for the fugacity and the $n-\beta p$ equation of state with series expansions.

We start with the cluster expansion of the grand partition function for $\mathrm{IRG}^{3}$ :

$$
\Xi(\beta, V, A)=\exp \left(\frac{V m^{2} g}{2 \pi^{2}} \sum_{k=1}^{\infty} \frac{A^{k}}{k^{2}}(-\eta)^{k-1} K_{2}(k m \beta)\right) .
$$

Now by using the relations (4a) and (4b) we get the pressure and particle density of the IRG as cluster expansions in terms of the normalized fugacity $z$,

$$
\begin{aligned}
& \beta p=L(m, \beta) \sum_{k=1}^{\infty} b_{k} z^{k}, \\
& n=L(m, \beta) \sum_{k=1}^{\infty} k b_{k} z^{k},
\end{aligned}
$$

where the cluster coefficients $b_{k}$ are defined as follows:

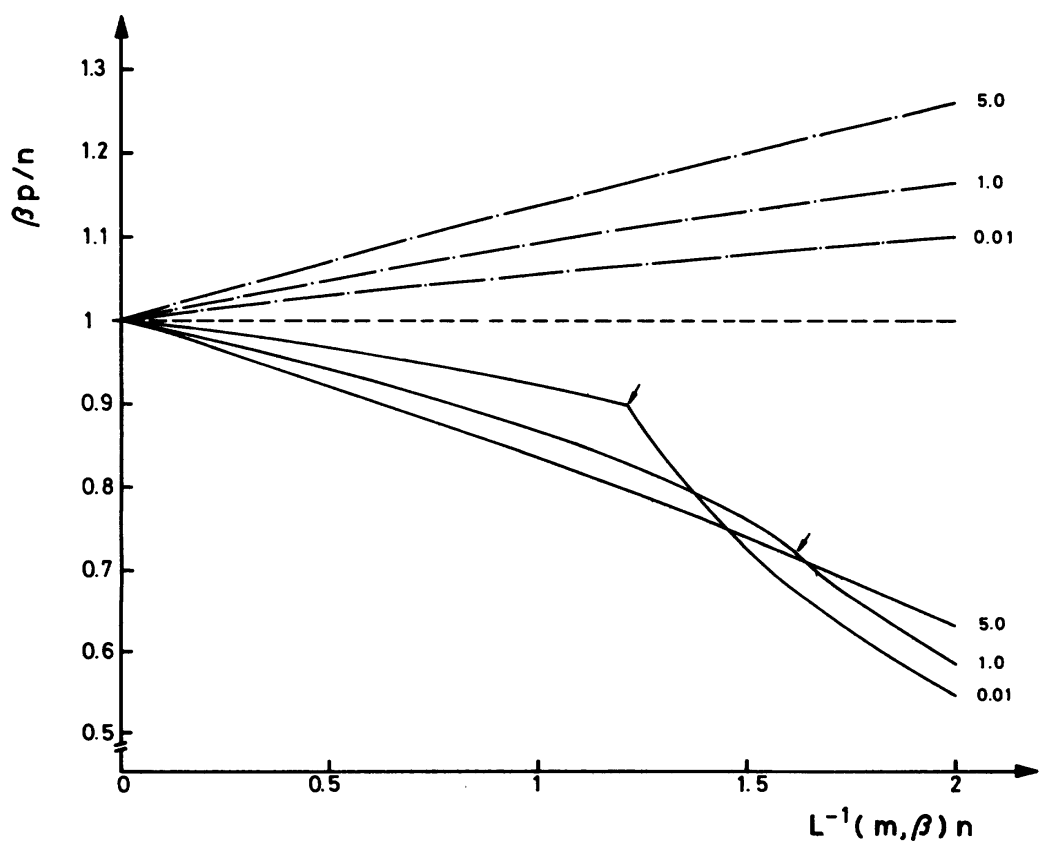

FIG. 3. Equation of state for relativistic Bose (-), Fermi (-- -), and Boltzmann (--) gases. 

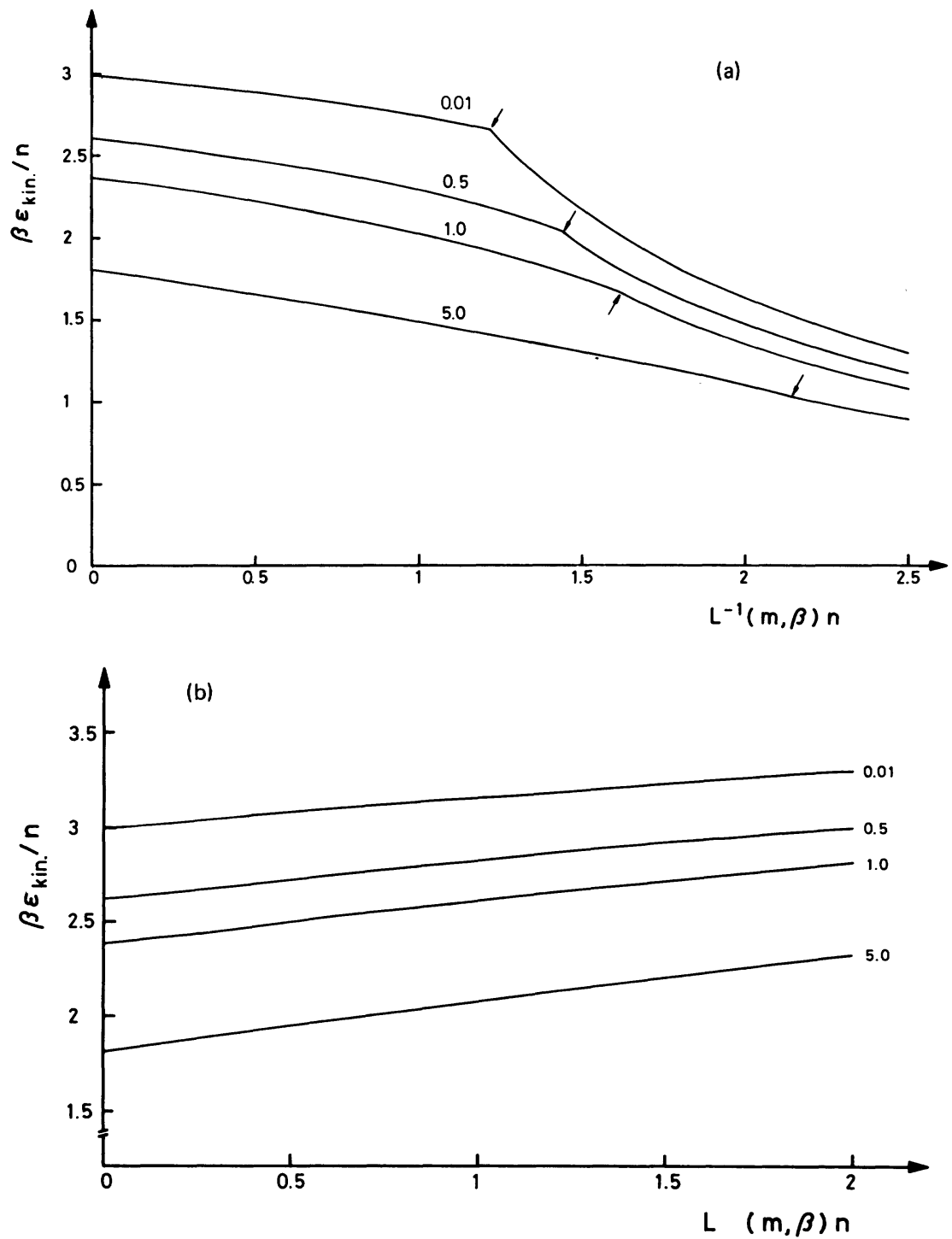

FIG. 4. Kinetic energy times inverse temperature per particle versus $L^{-1}(m, \beta) n$ for relativistic (a) Bose and (b) Fermi gases.

$$
b_{k}=\frac{(-\eta)^{k-1} K_{2}(k m \beta)}{k^{2} K_{2}(m \beta)} e^{(k-1) m \beta} .
$$

Here $b_{1}=1$ for all IRG, while for $k>1$ the nonvanishing cluster coefficients characterize the deviation of the relativistic quantum gases from their classical analog due to statistics. We notice that these corrections decrease monotonically with $m \beta$.

The virial expansion ${ }^{12}$ of the $n-\beta p$ equation of state can now be defined as

$$
\frac{\beta p}{n}=\sum_{j=1}^{\infty} a_{j}(\beta)\left[L^{-1}(m, \beta) n\right]^{j-1} \text {. }
$$

The coefficients $a_{j}(\beta)$ can be determined if one inserts Eqs. (20a), (20b) into (22) and compares both sides of the equation term by term. This substitution yields the well-known result $^{12}$

$$
\begin{aligned}
& a_{1}(\beta)=1, \\
& a_{2}(\beta)=-b_{2}, \\
& a_{3}(\beta)=4 b_{2}-2 b_{3}, \\
& a_{4}(\beta)=-20 b_{2}^{3}+18 b_{2} b_{3}-3 b_{4}, \\
& \vdots
\end{aligned}
$$

The contributions of different orders of the virial expansion (22) are compared with the exact solution (18a) in Fig. 5 for the case $m \beta=0.5$.

In order to discuss the connection between the exact solution and the virial expansion of the 


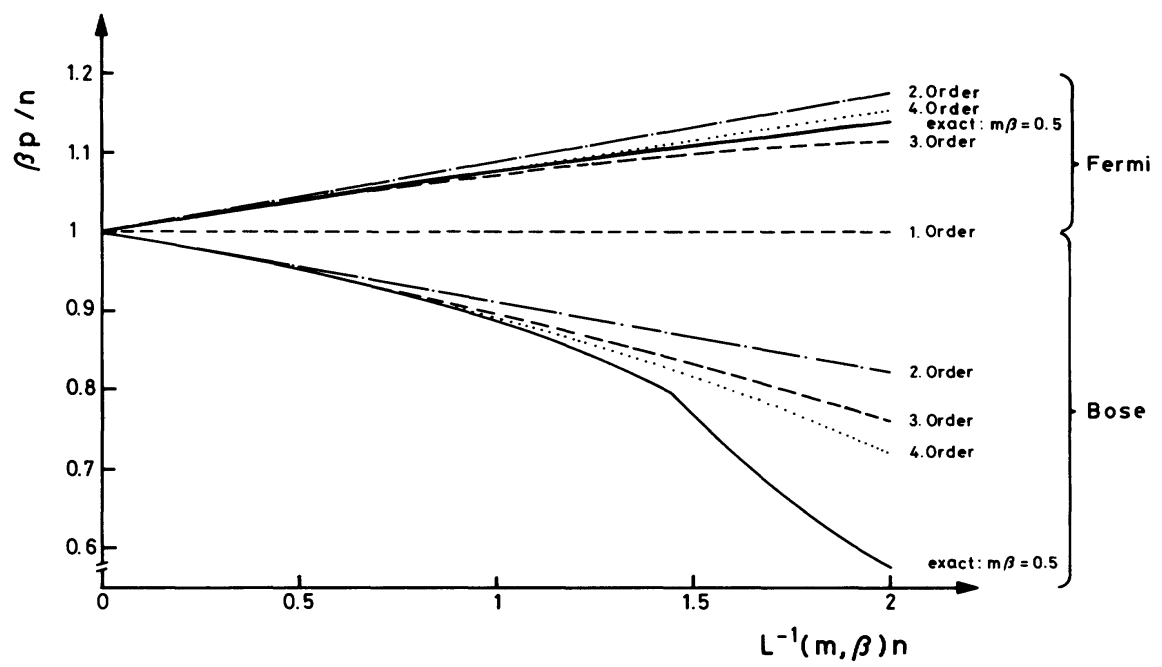

FIG. 5. Comparison between the exact equation of state for relativistic Bose and Fermi gases (-) and several orders of the corresponding virial expansions for the special value $m \beta=0.5$.

equation of state, we postulate the following expansions:

$$
\begin{aligned}
& -\eta-\frac{\eta}{\pi} \int_{1}^{\infty} d t \frac{\theta_{n, \eta}(t)}{t^{2}}=\sum_{j=1}^{\infty}\left[L^{-1}(m, \beta) n\right]^{j-1} d_{n, j}, \\
& -\eta-\frac{\eta}{\pi} \int_{1}^{\infty} d t \frac{\theta_{\ell, \eta}(t)}{t^{2}}=\sum_{j=1}^{\infty}\left[L^{-1}(m, \beta) \beta p\right]^{j-1} d_{p, j} .
\end{aligned}
$$

From these expressions we get a series expansion of the fugacity equations similar to the virial expansion for the equation of state

$$
\begin{aligned}
& \frac{1}{z}=\frac{1}{\beta p} \dot{L}(m, \beta)+\sum_{j=1}^{\infty}\left[L^{-1}(m, \beta) \beta p\right]^{j-1} d_{p, j}, \\
& \frac{1}{z}=\frac{1}{n} L(m, \beta)+\sum_{j=1}^{\infty}\left[L^{-1}(m, \beta) n\right]^{j-1} d_{n, j} .
\end{aligned}
$$

If we insert the expansion (25b) into the cluster expansion (20b), we get

$$
\begin{aligned}
n L^{-1}(m, \beta)=\sum_{k=1}^{\infty} & k b_{k}\left(1+\sum_{j=1}^{\infty}\left[L^{-1}(m, \beta) n\right]^{j} d_{n, j}\right)^{-k} \\
& \times\left[L^{-1}(m, \beta) n\right]^{k},
\end{aligned}
$$

from which we can determine the coefficients $d_{n, j}$ in terms of the cluster coefficients through the use of the geometric series expansion for the term in brackets. This evaluation is valid at low densities. We obtain

$$
\begin{aligned}
& d_{n, 1}=-2 b_{2}, \\
& d_{n, 2}=3 b_{3}-4 b_{2}^{2}, \\
& d_{n, 3}=4 b_{n}-18 b_{2} b_{3}+16 b_{2}^{3},
\end{aligned}
$$

Similarly, we can obtain the coefficients $d_{p, j}$ :

$$
\begin{aligned}
& d_{p, 1}=b_{2}, \\
& d_{p, 2}=b_{3}-b_{2}^{2}, \\
& d_{p, 3}=b_{4}-3 b_{2} b_{3}+2 b_{2}^{3},
\end{aligned}
$$

As an example we compare the first few orders of the expansion (25b) with the exact solution $(8 b)$ in Fig. 6. It should be noted that the higher-order corrections for the Fermi gas [Fig. 6(b)] yield better results for low particle densities but become worse than the first correction at higher densities. Of course, this could already be seen for the virial expansion, if we would have continued Fig. 5 to higher densities.

Whenever the fugacity expansions (25) are valid we get now a cluster expansion for the equation of state, which is symmetric in $\beta p$ and $n$ :

$$
\left(\frac{1}{n}-\frac{1}{\beta p}\right)=\sum_{j=1}^{\infty} L^{-j}(m, \beta)\left[(\beta p)^{j-1} d_{p, j}-n^{j-1} d_{n, j}\right] \text {. }
$$

From this expression one gets back the usual virial expansion if one solves for $\beta p / n$,

$$
\frac{\beta p}{n}=1-\beta p \sum_{j=1}^{\infty} L^{-j}(m, \beta)\left[n^{j-1} d_{n, j}-(\beta p)^{j-1} d_{p, j}\right],
$$

and then substitutes in $k$ th order the correction due to $(k-1)$ th order for $\beta p$. Thus we have found a connection between the exact equation of state and their virial expansion through the expansion of the 

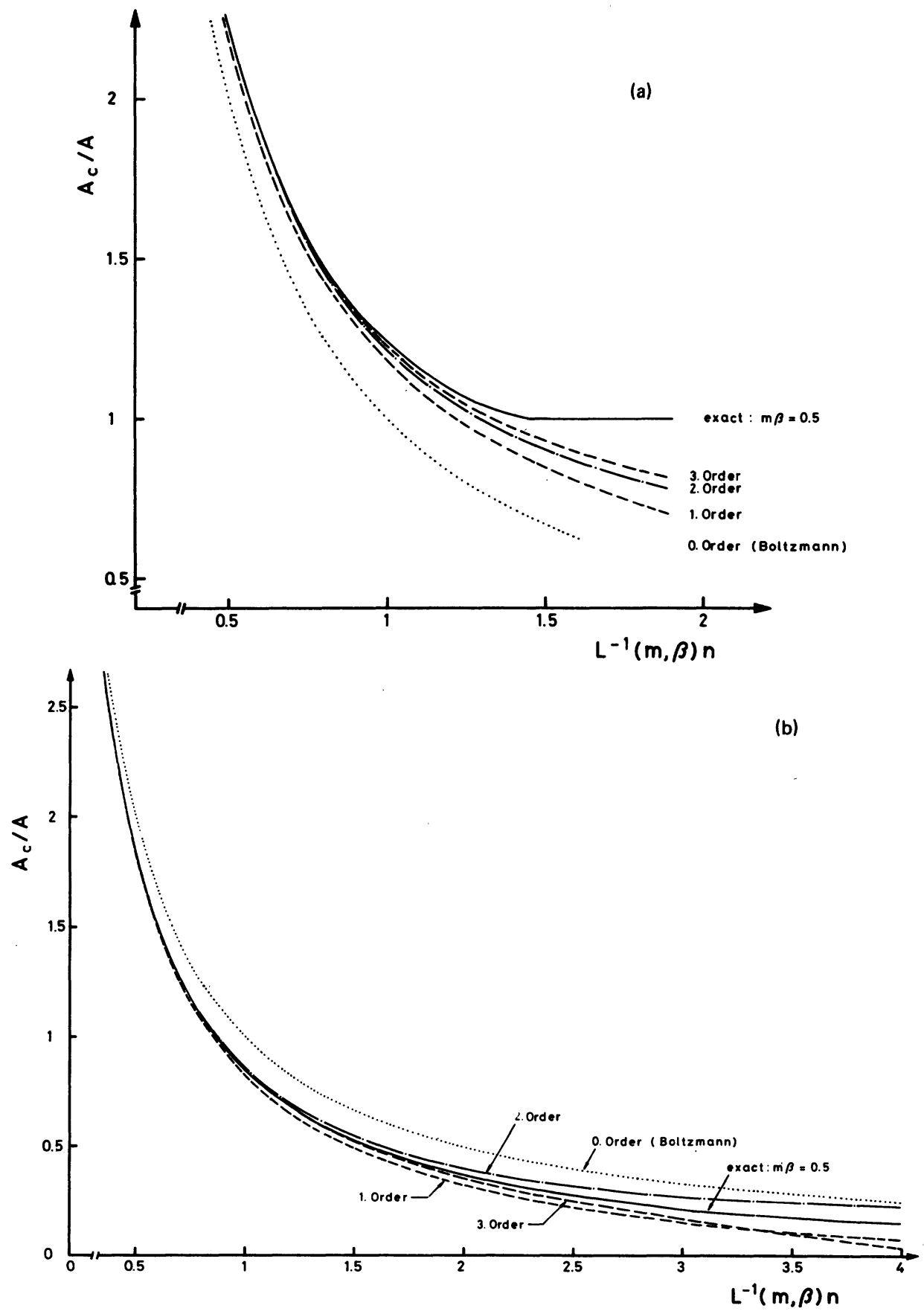

FIG. 6. Comparison between the exact solution (-) for the inverse normalized fugacity of (a) Bose and (b) Fermi gas and several orders of the corresponding series expansions for the special value $m \beta=0.5$.

statistic-dependent term of the exact fugacity equations.

\section{CONCLUSIONS AND GENERALIZATIONS}

In this paper we have directly computed the equations of state of the ideal relativistic quantum gases. Furthermore, we have shown how these equations are related to the series expansions in terms of the cluster coefficient. We shall now mention a few immediate generalizations of the ideal relativistic quantum gases. ${ }^{13}$

The extension of our calculations to a multicomponent system poses no additional formal problem for ideal gases. This is because the equilibrium conditions for a system with many different nonin- 
teracting particle types allow one to treat the components separately. This situation arises from the fact that the total grand partition function $\Xi$ is a simple product over the constitutent. Due to this fact the pressure as well as the density can be summed independently in terms of the constituent fugacities. Thus we obtain the equations for the constituents similar to (5a)-(5c) for $p_{i}, n_{i}$, and $\epsilon_{i}$, where $i$ labels different constituents as functions of $z_{i}$. Therefore we may repeat our analysis over again in connection with the cluster expansion as well as the virial expansion.

In order to simulate a strong interacting hadron gas one can introduce a mass spectrum of the form $\rho(m) \sim c m^{a} \exp (b m)$, where $a, b$, and $c$ are constants, as it results for example in the bootstrap model. ${ }^{14}$ Also in this case the foregoing procedure can be used to derive the exact equations of state. ${ }^{10}$ This is possible because the only difference arises from an additional integration over the mass spec- trum in Eqs. (7a)-(7c) and thus the one particle energy spectrum is not affected even in this extreme case of an interacting gas. The equations of state of a hadron gas enable one to study the relation of bootstrap to the statistics of a Bose gas, especially the connection between Bose condensation and the parameter $a$, which decides whether there is a highest temperature $\left(a \leqslant-\frac{7}{2}\right)$ or a phase transition $\left(a<-\frac{7}{2}\right)$ in the bootstrap model.

\section{ACKNOWLEDGMENTS}

We would like to thank $H$. Satz for discussions and his encouragement of this work. We are also very grateful to R. Beckmann for discussions, to J. Engels for advice on the computations as well as the use of his special programs, and to N. Bilic for looking at the manuscript. The financial support came from the Deutsche Forschungsgemeinschaft under Contract No. Mi 211/1.
${ }^{1}$ D. ter Haar and H. Wergeland, Phys. Rep. 1C, 31 (1971).

${ }^{2}$ B. Touschek, Nuovo Cimento 58B, 295 (1968). B. Touscheck and G. Rossi, Meccania Statistica (Boringhieri, Torino, 1970).

${ }^{3}$ M. Chaichian, R. Hagedorn, and M. Hayashi, Nucl. Phys. B92, 445 (1975).

${ }^{4} \mathrm{~J}$. L. Synge, The Relativistic Gas (North-Holland, Amsterdam, 1957).

${ }^{5}$ M. M. Nieto, Lett. Nuovo Cimento $\underline{1}, 677$ (1969); J. Math. Phys. 11, 1346 (1970).

${ }^{6}$ L. D. Landau and E. M. Lifshitz, A Course in Theore-

tical Physics (Pergamon, Oxford, 1969), Vol. V.

${ }^{7}$ A. Leonard, Phys. Rev. 175, 221 (1968).
${ }^{8}$ N. I. Muskehlishvili, Singuläre Integralgleichungen (Akademie, Berlin, 1965), 2nd ed.

${ }^{9} \mathrm{R}$. Balescu, Equilibrium and Non equilibrium Statistical Mechanics (Wiley, New York, 1975).

${ }^{10}$ F. Karsch and D. E. Miller, Phys. Lett. 79B, 259 (1978).

${ }^{11}$ M. Abramowitz and I. A. Stegun, Handbook of Mathematical Functions, 9th ed. (Dover, New York, 1970).

${ }^{12}$ A. Münster, Statistical Thermodynamics (Springer, Berlin, 1969), Vol. I.

${ }^{13}$ W. Israel, Ann. Phys. (N.Y.) 100,310 (1976); W. Israel and J. M. Stewart, ibid. $\overline{118}, 341$ (1979).

${ }^{14}$ R. Hagedorn, Nuovo Cimento Suppl. 3 , 147 (1965). 